\title{
Universal Motor Controller Designing for Washing Machines
}

\author{
David Margaria \\ Electrical Engineering Department \\ Zhejiang University, China
}

\author{
Afsoon Bahadoran \\ Computer Engineering Department \\ Azad University of Najafabad, \\ Esfahan, Iran
}

\begin{abstract}
The purpose of this paper is to obtain an accurate system model to test various control schemes for a motion control system that requires high speed, robustness and accuracy. A washing machine equipped with a universal motor is considered. The model of universal motor and its load (washing machine drum) based on the governing mathematical equations is developed in MATLABSIMULINK. Using the model, a PID and LQR controller to compensate the effects of disturbance without degrading tracking performance have been designed. In this experiment, it is not preferable for safety reasons to tune the controller online on the actual machinery. The aim of designing the mentioned controllers is rejection of unwanted disturbance caused by laundry position in washing machine; especially the major type of disturbance that occurs in transition mode. Finally, the simulation results of both controllers are compared.
\end{abstract}

Keywords: PID; LQR; Controller; Washing Machine; Universal Motor.

\section{INTRODUCTION}

In home appliances and light household equipment, electronics is commonly found in the man machine interface (dashboards, control panels, remote controls, etc...), as well as in the management of complex operating sequences, such as in washing machines; however, it is only just beginning to be used to control the electric motors which power them with high efficiency [1].

Energy saving, silence, flexibility and simplicity are requirements of growing importance: in applications such as drills, washing machines, vacuum-cleaners and refrigerators, variable speed control is the principal means of obtaining such performance features. Many motors are to be found in the various items of domestic equipment in a typical home. This illustrates the important role that electronics is bound to play in the control of home appliance motors.

Universal motors are widely used in household appliances like washing machine, vacuum cleaners, sewing machines and most domestic appliances because it is cost effective in respect of volume/power and it has a good torque response. In the control of these motors, generally, providing stable speed control, preventing large currents and drawing minimum harmonic current from ac mains supply are required. To meet these requirements using AC chopper with current and speed feedback is preferred. In addition, a control system with low cost is desired. To maintain the motor speed as the load varies, the application must control the voltage applied to the motor by either "phase angle modulation" techniques.

In the field of washing machine speed control the major challenge is inconstant applied torque to motor. Unpredictable place of laundry in washing machine results in disturbance of applied torque to motor. In addition, in transition mode from wash to dehydration (spin) change in position of laundry in drum results in sudden high changes in torque. Lee et. al tries to estimate the laundry moment of inertia in different washing stages in order to optimize the washing time by real time test equipments $[2,3]$.

Since, different types of motor are being utilized in washing machines, different controlling methods are reviewed for each motor type. Vaclavek and Blaha proposed Lyapanov function based for AC induction motor control Chi and $\mathrm{Xu}$ suggested sliding-mode $[4,5]$ sensorless control for direct drive washing machine motors $[6,7]$. In some other application fuzzy logic controlling method is used $[8,9]$. In this research, a universal motor is going to be controlled. Universal motors are the most common motors in washing machines because of low cost and high start torque. In this paper, both PID and LQR controlling are proposed for washing machine control [10].

This paper presents the experimental and theoretical studies of universal motors for washing machines. The mathematical model of the universal motor and its load (drum) in washing machine is formed at first and MATLAB-SIMULINK model is developed. This model uses the electromechanical parameters of the motor and drum. Using the model, a PID and LQR and adaptive controller [11] to compensate the effects of disturbance without degrading tracking performance have been designed. In this experiment, it is not preferable for safety reasons to tune the controller online on the actual machinery. Experimental results confirm that the model is a good approximation of washing machine dynamics and the proposed control methodology is effective. Finally, the simulation results of both controllers are compared.

\section{LOAD MODELING}

Main driving parts of washing machine includes motor, power transmission system (pulley \& shaft) and drum. Clothes in washing machine are washed via rotation of drum. All rotating parts are assembled in tub which is connected to washing machine structure by a suspension system which contains some springs and dampers. Figure 1 demonstrates washing machine main inner parts.

Each washing cycle of washing machine includes at least three main steps: washing, rinsing and spinning. The purpose of wash is removing dirt and soils from clothes. Therefore, in real time tests, clothes must be fallen from specific height in order to achieve maximum washing performance [12].

The purpose of rinsing cycle is removing detergent from clothes. Similarly, rinsing cycles requires $45 \mathrm{rpm}$ rotation speed. After wash and rinse mode washing machine starts 
dehydration mode. Due to the fact that dehydration cycle uses centrifugal force for removing wet from clothes, it requires high rotation speeds. Usual spinning speeds for dehydration is around $1000 \mathrm{rpm}$.
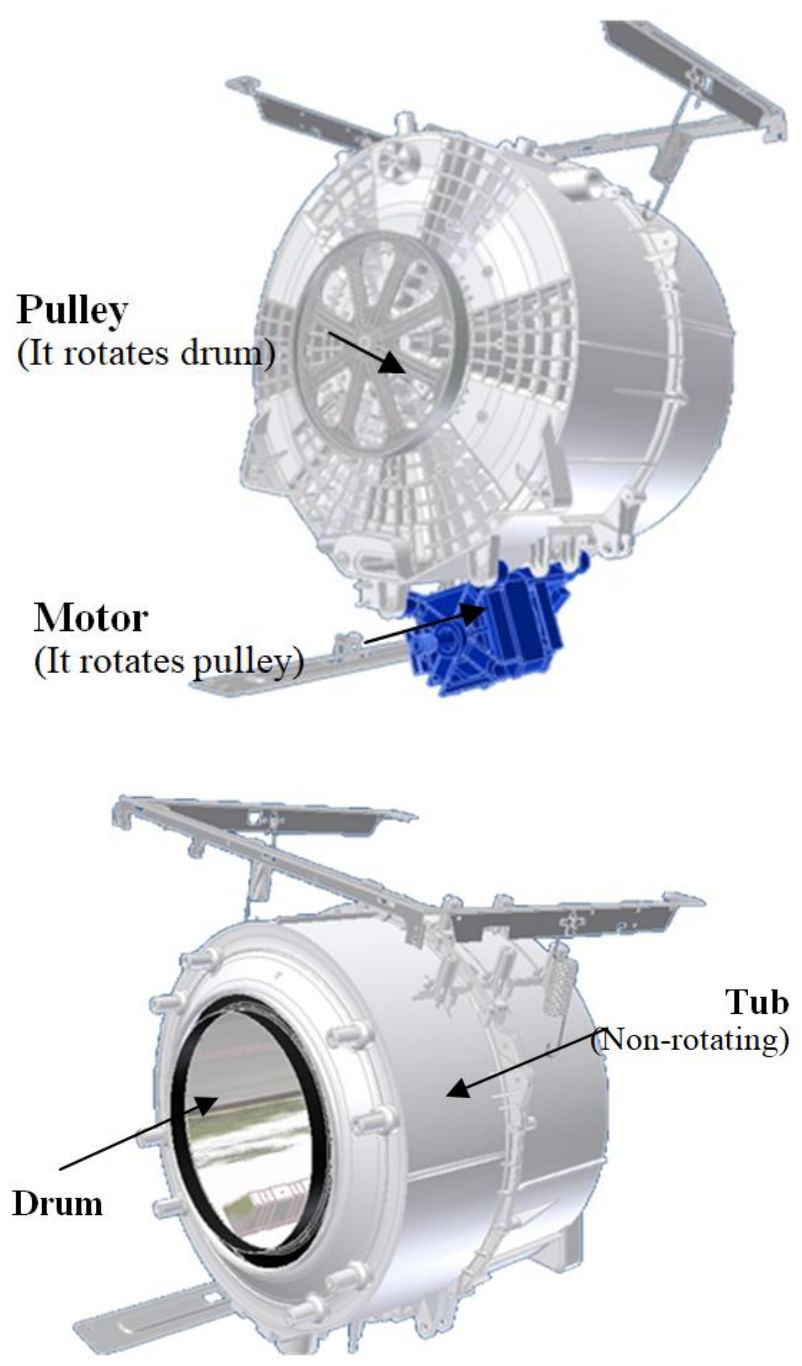

Figure 1. Inner parts of a horizontal washing machine.

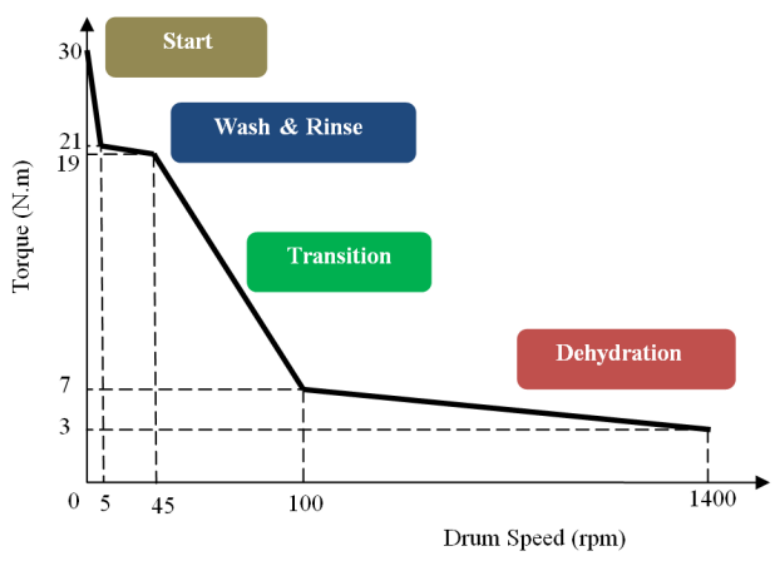

Figure 2. Typical diagram of torque changes in different modes of washing.
High viscosity between the drum and the laundry, force caused by falling laundry, and different moment of inertia are the main reasons which results in sudden torque changes while washing mode are changing to dehydration mode. Schematically, torque versus drum speed diagram is presented in Figure 2. Numbers in this figure are typical and differ regarding the amount of clothes in washing machine and washing machine structure. Therefore, a proper controller must be designed to reject the torque variation in the real time close loop of washing machine system.

Main challenges in motor speed control challenges are caused because of the following points $[13,14]$ :

1- Sudden torque changes in transition mode.

2- The exact mass of laundry in washing machine put by customer is not predictable. In other words, an operator may put 1 to $7 \mathrm{~kg}$ clothes in a $7 \mathrm{~kg}$ washing machine.

3- Exact position of laundry in washing machine drum cannot be determined.

\section{MATHEMATICAL MODEL}

The mathematical model of the universal motor and its load (drum) in washing machine is formed at first and MATLABSIMULINK model is developed. This model uses the electromechanical parameters of the motor and drum as follow. Figure 3 demonstrates mechanical model of universal motor in washing machine.

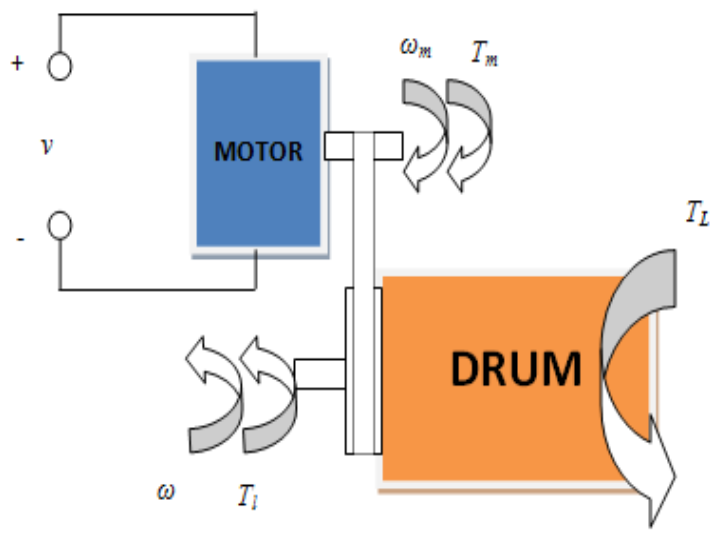

Figure 3- the schematic of motor and washing machine drum.

As shown in Figure 3, the motor and drum are linked by a belt and pulley. Applying Newton's second low to the rotor,

$$
\mathrm{J}_{\mathrm{m}} \dot{\omega}_{\mathrm{m}}=\mathrm{T}_{\mathrm{m}}-\mathrm{T}_{1}
$$

Where TL is the torque exerted on the motor shaft by the load, transmitted through the belt. So the torque at the motor shaft is seen to be the torque generated by the motor, minus the torque required to accelerate the motor. The torque exerted by the motor on the load shaft, transmitted through the pulley, is $\mathrm{NT}_{1}$. Newton's second low applied to the drum is

$$
\mathrm{J} \dot{\omega}=\mathrm{NT}_{\mathrm{m}}-\mathrm{T}_{\mathrm{L}}-\mathrm{NJ}_{\mathrm{m}} \dot{\omega}_{\mathrm{m}}
$$

Since , this becomes 
$\left(\mathrm{J}+\mathrm{N}^{2} \mathrm{~J}_{\mathrm{m}}\right) \dot{\omega}=\mathrm{NT}_{\mathrm{m}}-\mathrm{T}$

$\mathrm{J}_{\mathrm{e}}=\mathrm{J}+\mathrm{N}^{2} \mathrm{~J}_{\mathrm{m}}$

where $\mathrm{J}_{\mathrm{e}}$ is effective inertia seen at the load shaft.

$\mathrm{T}_{\mathrm{m}}=\mathrm{K}_{\mathrm{a}} \mathrm{i}_{\mathrm{a}}$

So

$\dot{\omega}=\frac{\mathrm{NK}_{\mathrm{a}}}{\mathrm{J}_{\mathrm{e}}} \mathrm{i}_{\mathrm{a}}-\frac{\mathrm{T}_{\mathrm{L}}}{\mathrm{J}_{\mathrm{e}}}$

To find the transfer function for the block diagram of the open and closed loop system a differential equation to describe the system dynamic. Kirchhoff's voltage is use to map the armature circuitry dynamic of the motor.

$\mathrm{L}_{\mathrm{a}} \frac{\mathrm{di}_{\mathrm{a}}}{\mathrm{dt}}+\mathrm{r}_{\mathrm{a}} \mathrm{i}_{\mathrm{a}}=\mathrm{v}-\mathrm{K}_{\mathrm{b}} \omega_{\mathrm{m}}$

From the transfer function, the block diagram of the motor and its load is illustrated by Figure 4 .

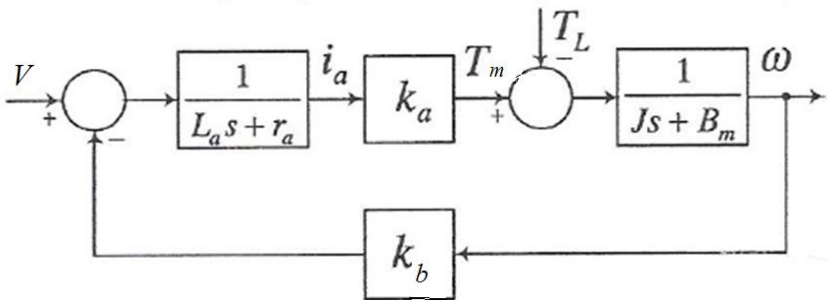

Figure 4. Block Diagram of the Universal Motor

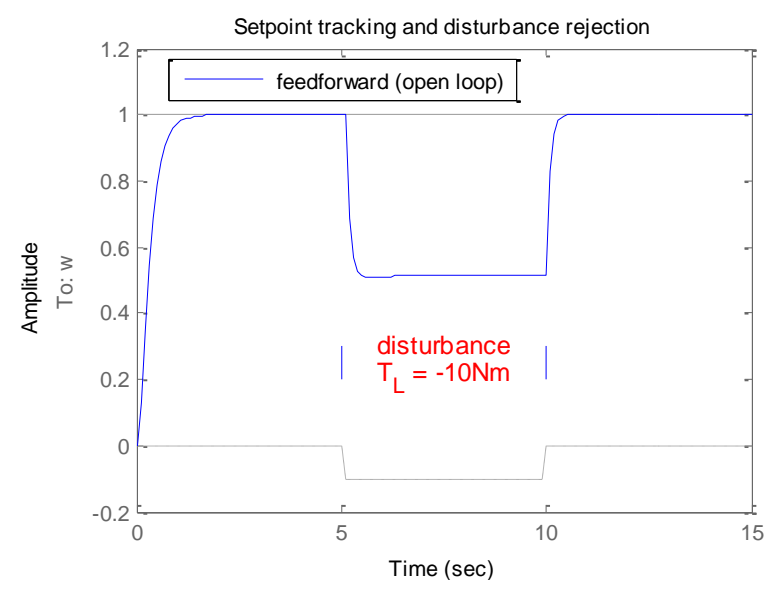

Figure 5. Open loop step response in peresence of a determined load $(10 \mathrm{Nm})$

To evaluate the feedforward design in the face of load disturbances, the response to a step command w_ref $=1$ with a disturbance $T_{d}=-10 \mathrm{Nm}$ between $\mathrm{t}=5$ and $\mathrm{t}=10$ seconds is depicted in Figure 5. This disturbance occurs in transition stage regarding to Figure 2. According to the response designing a proper controller for this system is inevitable.

\section{PID Controller}

PID Control (proportional-integral-derivative) is by far the widest type of automatic control used in industry. Even though it has a relatively simple algorithm/structure, there are many subtle variations[15] in how it is applied in industry. A proportional-integral-derivative controller (PID controller) is a generic control loop feedback mechanism widely used in industrial control systems [16, 17]. A PID controller will correct the error between the output and the desired input or set point by calculating and give an output of correction that will adjust the process accordingly. A PID controller has the general form

$$
v(t)=K_{p} e(t)+K_{i} \int_{0}^{t} e(\tau) d \tau+K_{d} \frac{d e}{d t}
$$

Where $K_{p}$ is proportional gain, $K_{i}$ is the integral gain, and $K_{d}$ is the derivative gain. The PID controller calculation (algorithm) involves three separate parameters; the Proportional, the Integral and Derivative values. The Proportional value determines the reaction to the current error, the Integral determines the reaction based on the sum of recent errors and the Derivative determines the reaction to the rate at which the error has been changing [16]. The weighted sum of these three actions is used to adjust the process via a control element such as the position of a control valve, the power supply of a heating element or universal speed and position through an intelligent hardware in the loop experiment setup [18].

Using the linear PID controller from the block diagram developed and documented in Figure 4, it obtains the closed loop system illustrated in Figure 6.

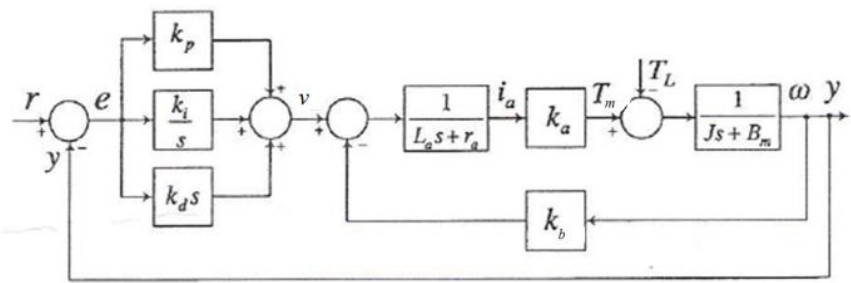

Figure 6. Block Diagram of the Closed-Loop Servo with PID Controller

\section{Linear Quadratic Regulator (LQR) Algorithm}

The advantages of used LQR are it is easy to design and increases the accuracy of the state variables by estimating the state. The nice feature of the LQR control as compared to pole placement is that instead of having to specify where $n$ eigenvalues should be placed a set of performance weighting are specified that could have more intuitive appeal. The result is a control that is guaranteed to be stable and the output of the circuit is very acceptable [19].

In layman's terms, Linear Quadratic Regulator (LQR) means the settings of a (regulating) controller governing either a machine or process (like an airplane or chemical reactor) are 
found by using a mathematical algorithm that minimizes a cost function with weighting factors supplied by a human (engineer). The "cost" (function) is often defined as a sum of the deviations of key measurements from their desired values. In effect this algorithm therefore finds those controller settings that minimize the undesired deviations, like deviations from desired altitude or process temperature. Often the magnitude of the control action itself is included in this sum as to keep the energy expended by the control action itself limited [20, 21].

In the particular case of a quadratic performance index combining the square of the error and square of the actuation, the solution to the optimal control problem is a feedback control where the measurements used for the feedback are all of the state variables. In this feedback control, each of the state variables is multiplied by a gain and the results are summed to get a single actuation value. The result of the LQR formulation is the set of gains, based on the relative weighting of the error and actuation in the performance index.

In addition to the integral of error, the LQR scheme also uses the state vector $\mathrm{x}=\left(\mathrm{i}_{\mathrm{a}}, \omega_{\mathrm{a}}\right)$ to synthesize the driving voltage $\mathrm{V}$, as shown in Figure 8. The resulting voltage is of the form

$$
\mathrm{v}=\mathrm{K}_{1} * \omega+\mathrm{K}_{2} * \omega / \mathrm{s}+\mathrm{K}_{3} * \mathrm{i}_{\mathrm{a}}
$$

For better disturbance rejection, a cost function that penalizes large integral error is used. The cost function is,

$C=\int_{0}^{\infty}\left(20 q(t)^{2}+\omega(t)^{2}+0.01 v(t)^{2}\right) d t$

$q(s)=\omega(s) / s$.

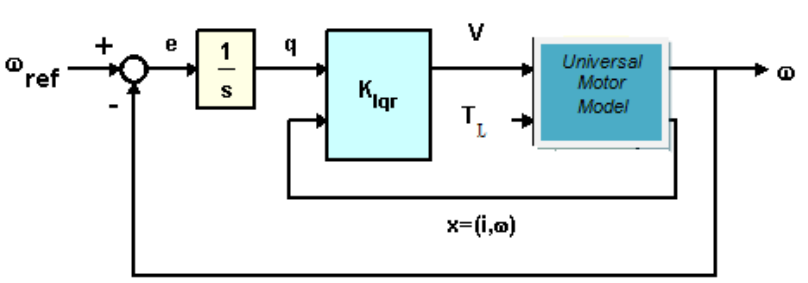

Figure 7. Block Diagram of the Closed-Loop Servo with LQR Controller

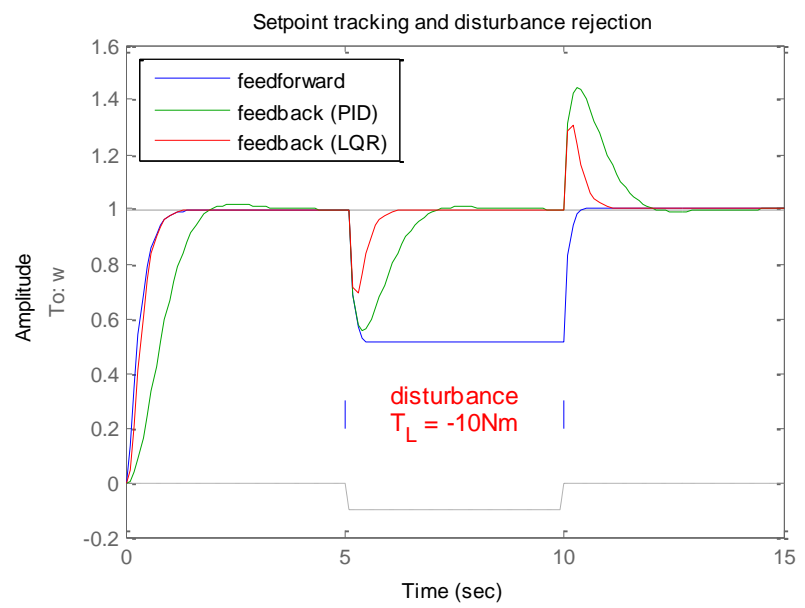

Figure 8. Open loop step response in peresence of a determined load $(10 \mathrm{Nm})$

To evaluate the PID and LQR controller design in the face of load disturbances, the response to a step command w_ref $=1$ with a disturbance $\mathrm{Td}=-10 \mathrm{Nm}$ between $\mathrm{t}=5$ and $\mathrm{t}=10$ seconds is simulated as shown in Figure 8.

\section{Conclusions}

In this paper, The model of universal motor and its load (washing machine drum) based on the governing mathematical equations is developed in MATLABSIMULINK. Then, a proper PID and LQR controller were proposed based on the desired response. To test these designed controller on unwanted disturbance caused by drum, a load exerted on system. The results as shown in Figure 8 , confirm that the PID controller cannot trace the set point as good as LQR controller can.

\section{Nomenclature}

$\begin{array}{ll}\mathrm{V} & \text { Voltage } \\ \omega \mathrm{m} & \text { Motor Speed } \\ \mathrm{Tm} & \text { Motor Torque } \\ \omega & \text { Drum Speed } \\ \mathrm{TL} & \text { Load } \\ \mathrm{ia} & \text { Current } \\ \mathrm{J} & \text { Drum moment of inertia } \\ \mathrm{Kb} & \text { Back emf constant } \\ \mathrm{Ka} & \text { Motor constant } \\ \mathrm{ra} & \text { Resistance } \\ \mathrm{La} & \text { Inductance } \\ \mathrm{Jm} & \text { Motor moment of inertia }\end{array}$

\section{REFERENCES}

[1] F. Rahmani, F. Razaghian, and A. Kashaninia, "Novel Approach to Design of a Class-EJ Power Amplifier Using High Power Technology," World Academy of Science, Engineering and Technology, International Journal of Electrical, Computer, Energetic, Electronic and Communication Engineering, vol. 9, pp. 541-546, 2015.

[2] J.-H. Lee, C.-H. Hwang, K.-m. Kim, W.C. Lee, C.-Y. Won, and Y.-R. Kim, "Optimal washing time control algorithm for the drum washing machine using an inertia estimator," in Power and Energy Conference, 2008. PECon 2008. IEEE 2nd International, 2008, pp. 1393-1398.

[3] S. Hashemi, M. Montazeri, and M. Nasiri, "The compensation of actuator delay for hardware-in-the-loop simulation of a jet engine fuel control unit," Simulation, vol. 90, pp. 745-755, 2014. 
[4] P. Vaclavek and P. Blaha, "Lyapunov function based flux and speed observer for ac induction motor sensorless control implementation on motorola dsp," in Industrial Technology, 2003 IEEE International Conference on, 2003, pp. 536-541.

[5] M. T. Andani and Z. Ramezani, "Robust Control of a Spherical Mobile Robot," 2017.

[6] S. Chi, Z. Zhang, and L. Xu, "Slidingmode sensorless control of direct-drive PM synchronous motors for washing machine applications," IEEE Transactions on Industry Applications, vol. 45, pp. 582590, 2009.

[7] K. Yousefpour, "Placement of Dispersed Generation with the Purpose of Losses Reduction and Voltage Profile Improvement in Distribution Networks Using Particle Swarm Optimization Algorithm," Journal of World's Electrical Engineering and Technology, vol. 2322, p. 5114, 2014.

[8] P. Guillemin, "Fuzzy logic applied to motor control," IEEE transactions on industry applications, vol. 32, pp. 51-56, 1996.

[9] H. Pourgharibshahi, M. Abdolzadeh, and

R. Fadaeinedjad, "Verification of computational optimum tilt angles of a photovoltaic module using an experimental photovoltaic system," Environmental Progress \& Sustainable Energy, vol. 34, pp. 1156-1165, 2015.

[10] M. T. Andani, H. Pourgharibshahi, Z. Ramezani, and H. Zargarzadeh, "Controller design for voltage-source converter using LQG/LTR," in Texas Power and Energy Conference (TPEC), 2018 IEEE, 2018, pp. 1-6.

[11] T. Pourseif, M. T. Andani, Z. Ramezani, and M. Pourgholi, "Model Reference Adaptive Control for Robot Tracking Problem: Design \& Performance Analysis," International Journal of Control Science and Engineering, vol. 7, pp. 18-23, 2017.

[12] B. Rahmani and S. R. Hashemi, "Internetbased control of FCU hardware-in-theloop simulators," Simulation Modelling Practice and Theory, vol. 56, pp. 69-81, 2015.

[13] A. Rouholamini, H. Pourgharibshahi, R. Fadaeinedjad, and G. Moschopoulos,
"Optimal tilt angle determination of photovoltaic panels and comparing of their mathematical model predictions to experimental data in Kerman," in Electrical and Computer Engineering (CCECE), 2013 26th Annual IEEE Canadian Conference on, 2013, pp. 1-4.

[14] M. Jafari, G. Atefi, J. Khalesi, and A. Soleymani, "A new conjugate heat transfer method to analyse a 3D steam cooled gas turbine blade with temperature-dependent material properties," Proceedings of the Institution of Mechanical Engineers, Part C: Journal of Mechanical Engineering Science, vol. 226, pp. 1309-1320, 2012.

[15] A. Mohammed, M. Alhadri, W. Zakri, and H. Aliniagerdroudbari, "Design and Comparison of Cooling Plates for a Prismatic Lithium-ion Battery for Electrified Vehicles," SAE Technical Paper, pp. 01-1188, 2018.

[16] J. Tang, "PID controller using the TMS320C31 DSK with online parameter adjustment for real-time DC motor speed and position control," in Industrial Electronics, 2001. Proceedings. ISIE 2001. IEEE International Symposium on, 2001, pp. 786-791.

[17] M. H. Imani, K. Yousefpour, M. J. Ghadi, and M. T. Andani, "Simultaneous presence of wind farm and V2G in security constrained unit commitment problem considering uncertainty of wind generation," in Texas Power and Energy Conference (TPEC), 2018 IEEE, 2018, pp. 1-6.

[18] S. R. Hashemi and M. Montazeri-Gh, "Polynomial-based time-delay compensation for hardware-in-the-loop simulation of a jet engine fuel control unit," International Journal of Automation and Control, vol. 8, pp. 323-338, 2014.

[19] F. Rahmani, F. Razaghian, and A. Kashaninia, "High Power Two-Stage Class-AB/J Power Amplifier with High Gain and Efficiency," Journal of Academic and Applied Studies (JAAS), vol. 4, pp. 56-68, 2014.

[20] R. C. Nelson, Flight stability and automatic control vol. 2: WCB/McGraw Hill New York, 1998.

[21] B. Etkin and L. D. Reid, Dynamics of flight: stability and control vol. 3: Wiley New York, 1996. 